\title{
1 Human fecal microbiota is associated with colorectal cancer
}

3 Authors

4 Qiulin Yao $^{1 *}$, Meifang Tang ${ }^{2,1^{*}}$, Liuhong Zeng ${ }^{1}$, Zhonghua $\mathrm{Chu}^{3}$, Hui Sheng ${ }^{4}$, Yuyu

5 Zhang ${ }^{5}$, Yuan Zhou ${ }^{1}$, Hongyun Zhang ${ }^{1}$, Huayan Jiang ${ }^{5}$, Mingzhi Ye $e^{6,1,5 \#}$

6

\section{Affiliations}

$8{ }^{1}$ Clinical laboratory of BGI Health, BGI-Shenzhen, Shenzhen 518083, China

$9 \quad{ }^{2}$ BGI Education Center, University of Chinese Academy of Sciences, Shenzhen

$10 \quad 518083$, China

$11{ }^{3}$ Guangdong Provincial Key Laboratory of Malignant Tumor Epigenetics and Gene

12 Regulation, Department of Gastrointestinal Surgery, Sun Yat-sen Memorial Hospital,

13 Sun Yat-sen University, Guangzhou 510060, China

$14{ }^{4}$ Department of Experimental Research, State Key Laboratory of Oncology in South

15 China, Collaborative Innovation Center for Cancer Medicine, Sun Yat-Sen University

16 Cancer Center, Guangzhou 510060, China

$17{ }^{5}$ BGI Genomics, BGI-Shenzhen, Shenzhen 518083, China

$18{ }^{6}$ BGI-Guangzhou Medical Laboratory, BGI-Shenzhen, Guangzhou 510006, China

$20 *$ These authors contributed equally to the work

21 \# Address correspondence to: Mingzhi Ye, PHD

22 Zone B Room 401, Qinglan Street, Panyu District, Guangzhou 510006, China 
E-mail: yemingzhi@genomics.cn

25 Abstract

26 Background: Colorectal cancer (CRC) is one of the most common cancers. In recent

27 studies, the gut microbiota has been reported to be potentially involved in aggravating

28 or favoring CRC development. However, little is known about the microbiota

29 composition in CRC patients after treatment. In this study, we explored the fecal

30 microbiota composition to obtain a periscopic view of gut microbial communities. We

31 analyzed microbial 16S rRNA genes from 107 fecal samples of Chinese individuals

32 from three groups, including 33 healthy individuals (Normal), $38 \mathrm{CRC}$ patients $(\mathrm{Fa})$,

33 and $36 \mathrm{CRC}$ post-surgery patients $(\mathrm{Fb})$.

34 Results: Species richness and diversity were decreased in the $\mathrm{Fa}$ and $\mathrm{Fb}$ groups

35 compared with that of the Normal group. Partial least squares discrimination analysis

36 showed clustering of samples according to disease with an obvious separation

37 between the $\mathrm{Fa}$ and Normal, and $\mathrm{Fb}$ and Normal groups, as well as a partial separation

38 between the $\mathrm{Fa}$ and $\mathrm{Fb}$ groups. Based on linear discriminant analysis effect size

39 analysis and a receiver operating characteristic model, Fusobacterium was suggested

40 as a potential biomarker for CRC screening. Additionally, we found that surgery

41 greatly reduced the bacterial diversity of microbiota in CRC patients. Some

42 commensal beneficial bacteria of the intestinal canal, such as Faecalibacterium and

43 Prevotella, were decreased, whereas the drug-resistant Enterococcus was visibly

44 increased in CRC post-surgery group. Meanwhile, we observed a declining tendency 
of Fusobacterium in the majority of follow-up CRC patients who were still alive

46 approximately 3 y after surgery. We also observed that beneficial bacteria

47 dramatically decreased in CRC patients that recidivated or died after surgery. This

48 revealed that important bacteria might be associated with prognosis.

49 Conclusions: The fecal bacterial diversity was diminished in CRC patients compared

50 with that in healthy individuals. Enrichment and depletion of several bacterial strains

51 associated with carcinomas and inflammation were detected in CRC samples.

52 Fusobacterium might be a potential biomarker for early screening of CRC in Chinese

53 or Asian populations. In summary, this study indicated that fecal microbiome-based

54 approaches could be a feasible method for detecting CRC and monitoring prognosis

55 post-surgery.

57 Keywords: Colorectal cancer, 16s rRNA gene sequencing, Fusobacterium,

58 Faecalibacterium, Prevotella, biomarker.

\section{Introduction}

63 Because of its high incidence, increased difficulty in early diagnosis, and high

64 mortality rate, colorectal cancer has become a major public health issue,

65 especially in less developed regions. Moreover, survival and risk of recurrence

66 have been reported to vary based on the stage of the tumor. According to the 

surgery can be curative with a 5 -y survival rate of up to $80 \%$; however, the prognosis is dramatically decreased in tumors at a later stage, due to the increased occurrence of metastasis ${ }^{3}$. Therefore, a new diagnostic method for the early detection of lesions that would be noninvasive and easy to perform, is gaining attention among researchers.

Gut microbiota have been suggested to be potentially involved in the development of colorectal cancer. Bacteria and their related products might participate in the initiation or progression of sporadic colorectal cancer by a variety of mechanisms, including induction of inflammation, production of

83 to be associated with colonic neoplasia ${ }^{6}$. The colibactin-producing E. coli has been

84 reported to directly attack the host DNA, by introducing DNA breaks that lead to 85 genomic instability and increased mutation frequency ${ }^{4,7}$. Whereas, Enterococcus 86 faecalis is known to indirectly lead to DNA damage in the epithelium by inducing high levels of $\operatorname{ROS}^{8,9}$, which are typically produced by host cells during inflammation. Fusobacterium nucleatum has been reported to modulate the 
89 tumor-immune microenvironment, potentiating intestinal tumorigenesis in mice ${ }^{10}$.

90 In addition, some studies have indicated that $F$. nucleatum is enriched in the gut of

91 CRC patients ${ }^{11,12}$ and have even suggested it as a putative prognostic factor in $92 \mathrm{CRC}^{13,14,15}$.

93 Changes in the abundance of some gut commensal bacteria have been linked

94 to dysbiosis observed in several human diseases. One such case regards 95 Faecalibacterium prausnitzii, a protective bacterium, which was found to be 96 decreased in CRC patients ${ }^{6}$. Culture supernatants of $F$. prausnitzii were shown to 97 protect mice against 2,4,6-trinitrobenzenesulfonic acid-induced colitis, a potent 98 risk factor for colon cancer ${ }^{16}$. The collection of fecal samples, in which the 99 microorganism composition is known to be highly correlated with the colonic 100 lumen and mucosa, seems to be an ideal approach, as this data can provide a 101 periscopic view of gut microbial communities ${ }^{17}$, without the need for invasive 102 procedures, such as colonoscopies.

103 To date, a large amount of research has focused on the gut microbiota of 104 CRC patients; however, the microenvironmental changes in the colorectum of 105 patients after therapy, such as surgery, chemotherapy, or radiotherapy, have not 106 been widely studied. As specific bacteria might drive tumorigenesis, we aimed to 107 identify whether the population of these bacteria were decreased after effective 108 treatment. If so, it would indicate that effective treatment might result in the 109 alteration of the microbiota of CRC patients to one more similar to that of normal 110 samples. 
111 Thus, to understand the structure of the gut microbial community and the

112 changes post-surgery in CRC patients, we investigated the microbiota in the stools

113 of CRC patients, CRC patients after surgery, and healthy individuals using $16 \mathrm{~S}$

114 rDNA amplicon sequencing.

115

116

117

2. Results

Summary of the study

Our study population was composed of 33 healthy individuals (Normal), 38

\begin{tabular}{cccc}
\hline & Fa & Fb & Normal \\
\hline Number & 38 & 36 & 33 \\
Male, $\mathbf{n}(\%)$ & $24(63.16)$ & $23(63.89)$ & $17(51.52)$ \\
Mean age ( $\mathbf{H}$ SD, y) & $64.32 \pm 11.14$ & $63.19 \pm 10.73$ & $59.00 \pm 4.94$ \\
Pathological stage & & & \\
I/II/III (\%) & $13.51 / 35.14 / 51.35$ & $8.82 / 44.12 / 47.06$ & - \\
\hline
\end{tabular}

124 We obtained a total of 4992311 16S rDNA sequences from 107 stool samples, with an average of $46657 \pm 2955$ reads per sample in the whole cohort. 
128 Normal, Fa, and Fb groups, respectively. The maximum number of OTUs for a

129 single sample was 401, whereas the minimum, which was found in the $\mathrm{Fb}$ group,

130 was only 27 (Table S1). There were 495 common OTUs in all groups, with the Fa

131 group having the most specific OTUs, whereas the $\mathrm{Fb}$ group having the least

132 specific OTUs (Figure S1).

\section{Richness and diversity}

The observed species and Chao richness index, Shannon, and Simpson

136 diversity index were used to describe the alpha diversity features of the bacterial

137 communities in our samples. We observed that the species richness and diversity

138 in $\mathrm{Fa}$ and $\mathrm{Fb}$ were decreased compared with those in the Normal group. A strong

139 decrease in biodiversity was observed in the $\mathrm{Fb}$ group, especially in the stages II

140 and III subgroup of Fb, compared with that in the other groups (Figures 1A, S2).

141 We used analysis of similarities (ANOSIM) to estimate the similarity among

142 groups. Our results indicated that differences among groups were more significant

143 than differences within groups $(\mathrm{R}$-value $=0.164, \mathrm{P}=0.001)($ Figure 1B). At the

144 same time, a beta diversity evaluation, represented by partial least squares

145 discrimination analysis (PLSDA), showed a clustering of samples according to

146 disease with an obvious separation between the Fa and Normal groups, and the Fb

147 and Normal groups, but a partial separation between the $\mathrm{Fa}$ and $\mathrm{Fb}$ groups.

148 Permutational multivariate analysis of variance confirmed this observation 

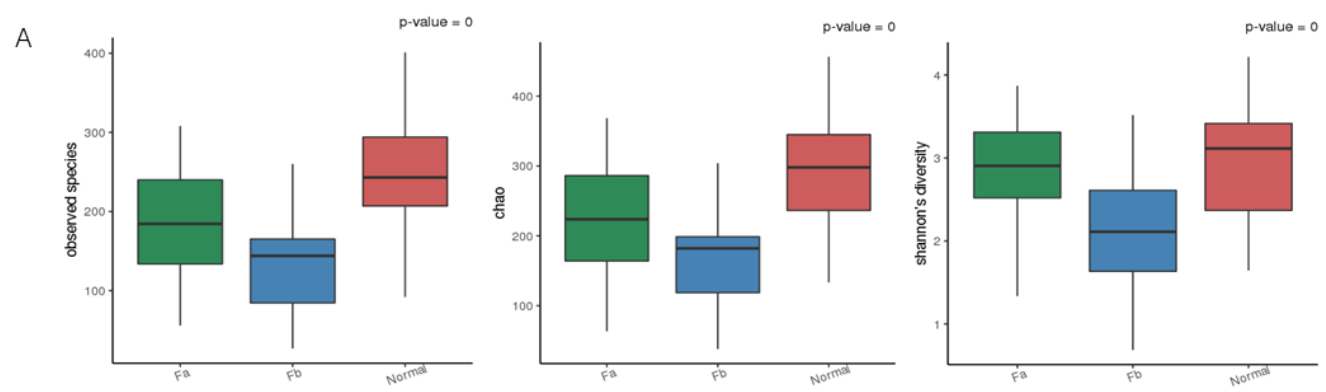

B

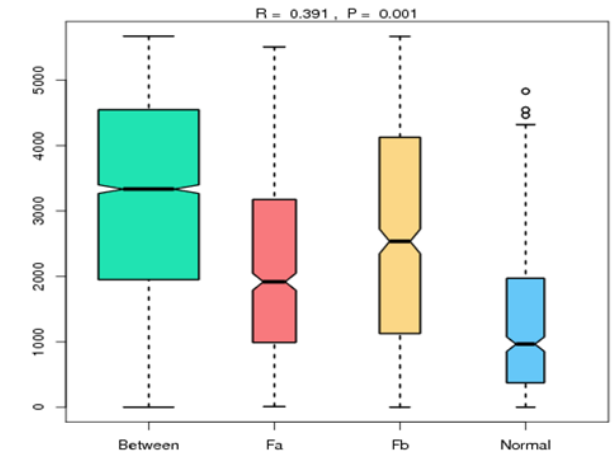

C

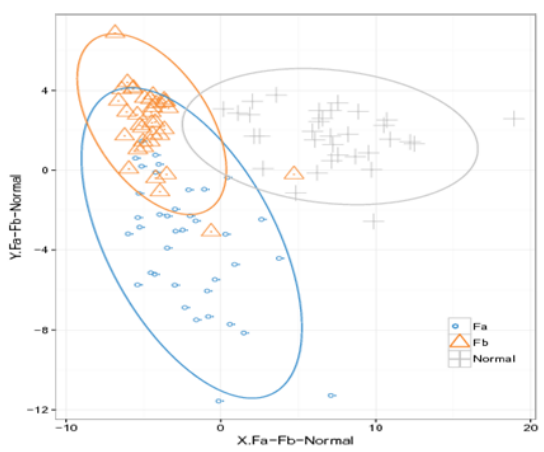

Figure 1. Microbiota biodiversity. (A) Alpha diversity. Bigger indexes of the observed species and Chao reflect greater richness, whereas a bigger Shannon index reflects greater diversity. $\mathrm{R}^{2}=0.18593, \mathrm{P}=0.001(* * *)$.

\section{Bacterial microbiota composition}

We analyzed the composition and abundance of bacteria at all taxonomic 
164 levels. As expected, we found that a large majority of the bacteria in the $\mathrm{Fa}, \mathrm{Fb}$,

165 and normal samples belonged to the phyla Bacteroidetes, Firmicutes,

166 Proteobacteria, Fusobacteria, and Actinobacteria. We further identified that the

167 distribution of the major phyla in the Normal group was consistent with published

168 data. Further comparison of the relative abundance revealed clear differences. The

169 most abundant phylum in the Normal group was Bacteroidetes, followed by

170 Firmicutes, Proteobacteria, and Actinobacteria. However, an increase in the

171 distribution of Proteobacteria, Fusobacteria, and Verrucomicrobia was observed in

172 the Fa group. Except for an increase in the distribution of Fusobacteria and

173 Verrucomicrobia, the $\mathrm{Fb}$ group was characterized by a notable increase in

174 Proteobacteria and a decrease in Firmicutes (Figure S3).

175 We identified a total of 173 genera at the genus level, with the dominant 176 genus among all groups being Bacteroides (34.89\%, $32.34 \%$, and $22.89 \%$ in 177 Normal, Fa, and Fb groups, respectively). However, apart from this, the 178 composition and prevalence of genera was different among the three groups. In 179 the Normal group, we identified the following genera: Prevotella (21.49\%), 180 Faecalibacterium (8.58 \%), Roseburia (3.28\%), and Ruminococcus (3.02\%). The 181 Fa group was characterized by the presence of Escherichia (10.69\%), 182 Faecalibacterium (5.49\%), Prevotella (4.78\%), and Parabacteroides (3.84 \%); 183 whereas in the $\mathrm{Fb}$ group, we observed Escherichia (18.56 \%), Parabacteroides 184 (6.81 \%), Enterococcus (5.82\%), and Morganella (4.68 \%) (Table S2). 185 Accordingly, Escherichia belonging to the phylum Proteobacteria, Fusobacterium 
186 belonging to Fusobacteria, and Parabacteroides were found to be enriched in

187 CRC patients ( $\mathrm{Fa}$ and $\mathrm{Fb}$ groups) compared with that in the Normal group,

188 whereas Prevotella was demonstrated to be overrepresented in the Normal group.

189 The presence of Faecalibacterium was scarce, whereas Enterococcus was

190 abundant in the Fb group (Figure 2). Although Bacteroides exhibited a similar

191 relative abundance at the genus level, at the species level, the abundance of $B$.

192 fragilis was shown to vary among groups (Figure S4), being enriched in the Fa

193 and $\mathrm{Fb}$ groups.

194

195

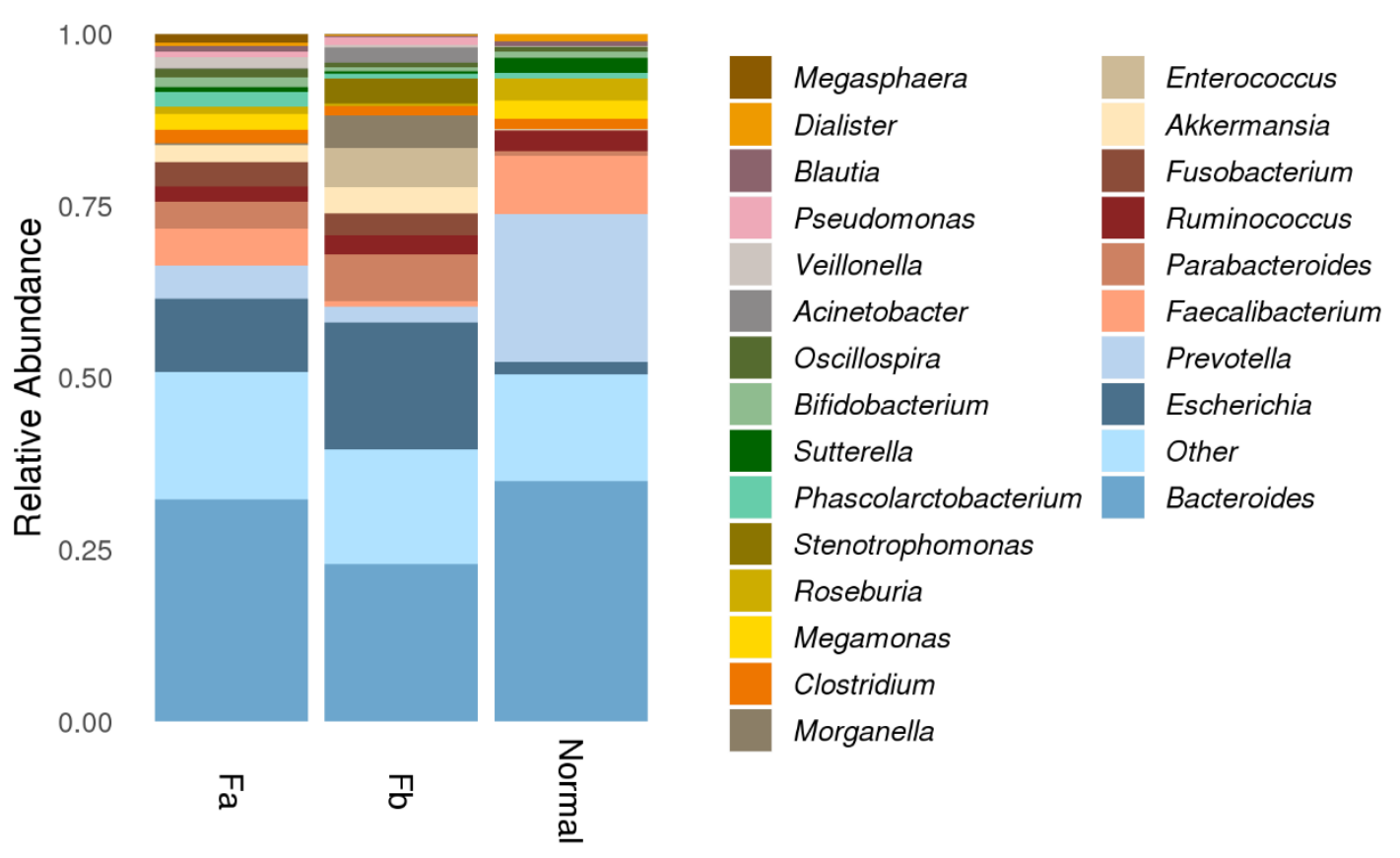

Figure 2. Microbiota composition in every group at the genus level. Relative abundances of less than $0.5 \%$ were combined and shown as other.

$\mathrm{Fa}, \mathrm{CRC}$ patients before treatment; $\mathrm{Fb}, \mathrm{CRC}$ patients after surgery. 

used the linear discriminant analysis (LDA) effect size (LEfSe) biomarker biomarkers showing statistical differences. We performed LEfSe analysis at both the family and genus levels, and found 52 discriminative features using a threshold of LDA score of $2(\mathrm{P}$ value $<0.01)$ at the genus level (Table S3). We observed that Prevotella $(\mathrm{LDA}=4.95, \mathrm{P}<0.01)$, Faecalibacterium $(\mathrm{LDA}=4.66$, $\mathrm{P}<0.001)$, Roseburia $(\mathrm{LDA}=4.23, \mathrm{P}<0.001)$, Megamonas $(\mathrm{LDA}=4.21, \mathrm{P}<$ $0.001)$, and Sutterella $(\mathrm{LDA}=4.08, \mathrm{P}<0.001)$ were the dominant microbes in the Normal group; Fusobacterium $(\mathrm{LDA}=4.22, \mathrm{P}<0.001)$ was the dominant genus

211 in the Fa group, whereas Escherichia $(\mathrm{LDA}=4.96, \mathrm{P}<0.001)$, Enterococcus

$212(\mathrm{LDA}=4.49, \mathrm{P}<0.001)$, and Stenotrophomonas $(\mathrm{LDA}=4.17, \mathrm{P}<0.01)$ were the

213 dominant genera in the Fb group. The genera with an LDA score higher than 3 are

214 displayed in Figure 3A. To further compare the relative abundance of these

215 primary biomarkers in all groups, we evaluated the average relative abundance of

216 bacteria with an LDA score higher than 4 in every group. Except for Bacteroides,

217 all other bacteria showed significant differences $(\mathrm{P}<0.01)($ Figure 3B$)$.

218 Consecutively, to explore whether these differential microbes were suitable 219 for CRC detection, or classification of CRC samples before or after treatment, we 220 used the receiver operating characteristic (ROC) curve to evaluate their predictive 221 power. First, we calculated the area under the ROC curve (AUC) of the microbes between the Fa and Normal groups, and found that the most discriminative genus 

previous studies suggesting Fusobacterium as prevalent in the gut of CRC patients ${ }^{11,12}$, potentially accelerating tumorigenesis ${ }^{10}$, our results further confirmed this enrichment and indicated a potential biomarker for detecting CRC. Following,
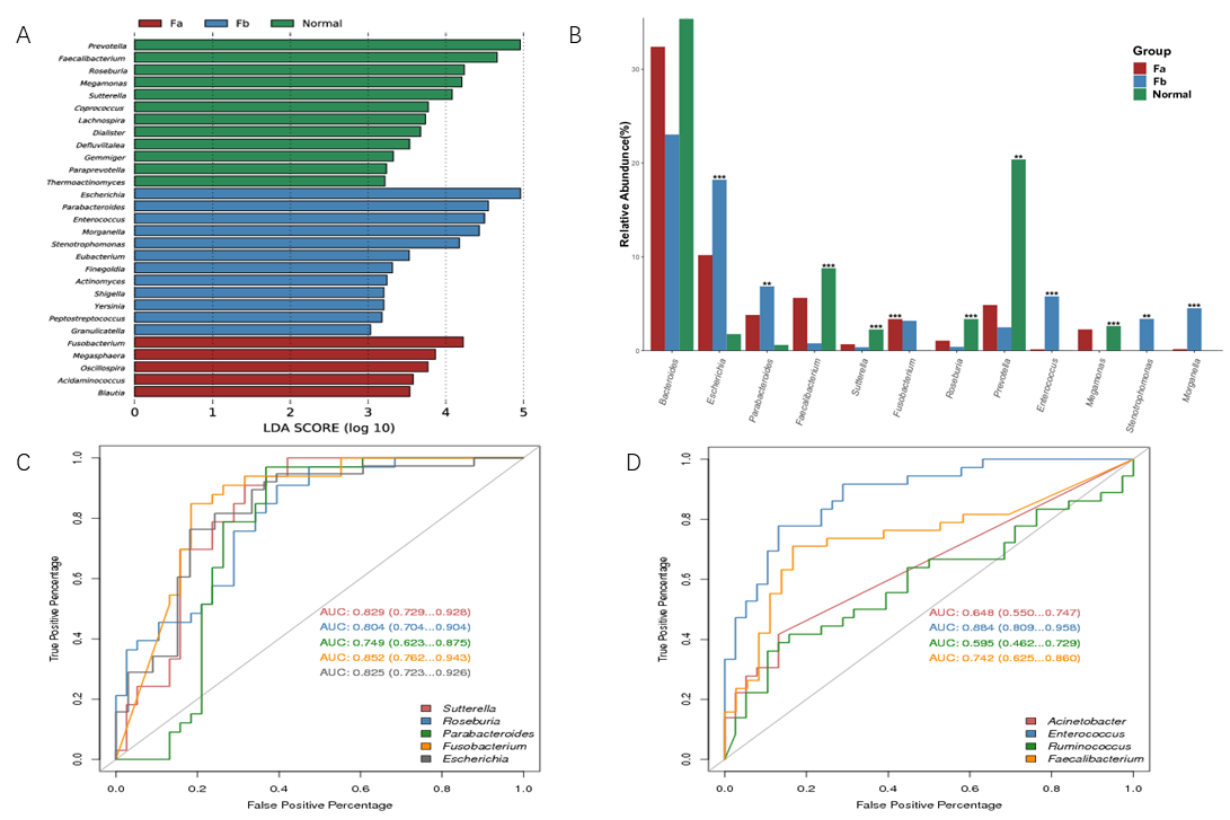

Figure 3. (A) LEfSe analysis for taxonomic biomarkers on the genus level among the three

groups. Each color represents one group. (B) Differential comparison of key microbes. ${ }^{* * *}, \mathrm{P}$ 
238 LEfSe, linear discriminant analysis effect size; ROC, receiver operating characteristic; AUC,

239 area under the ROC curve; Fa, CRC patients before treatment; Fb, CRC patients after surgery.

241 Function analysis and correlation with clinic data

242 We used Picrust2 to predict the MetaCyc pathways of microbiota in every

243 sample. This analysis revealed the differential functions between CRC patients

244 and healthy individuals and between CRC patients before and after treatment. We

245 observed that the pathways enriched in the Fa group compared with those of the

246 Normal group were those of lipid, fatty acid, amino acid, aldehyde, alcohol, and

247 aromatic compound degradation (Figure 4A). Only the metabolic regulator

248 biosynthesis as well as fatty acid and lipid degradation pathways were identified

249 to be significantly $(\mathrm{P}<0.01,|\log 2 \mathrm{FC}|>1)$ different in the $\mathrm{Fa}$ and $\mathrm{Fb}$ groups.

$250 \quad$ (Figure 4B).

We collected 56 clinical indexes of CRC patients, including biochemical criteria and routine blood examinations. At the genus level, 27 differentially abundant microbes (LDA score $>3, \mathrm{P}<0.01$ ) were selected and related to the

254 clinical data. We calculated the Pearson coefficient of pairwise correlation 255 between microbes and clinical indexes, and characters exhibiting a high 256 correlation (Pearson coefficient $\geq 0.7$ ) are displayed on a heatmap (Figure 4C). A 257 similar abundance model and very strong correlation could be observed in some 258 microbes, such as Escherichia, Enterococcus, Yersinia, and Eubacterium, which 

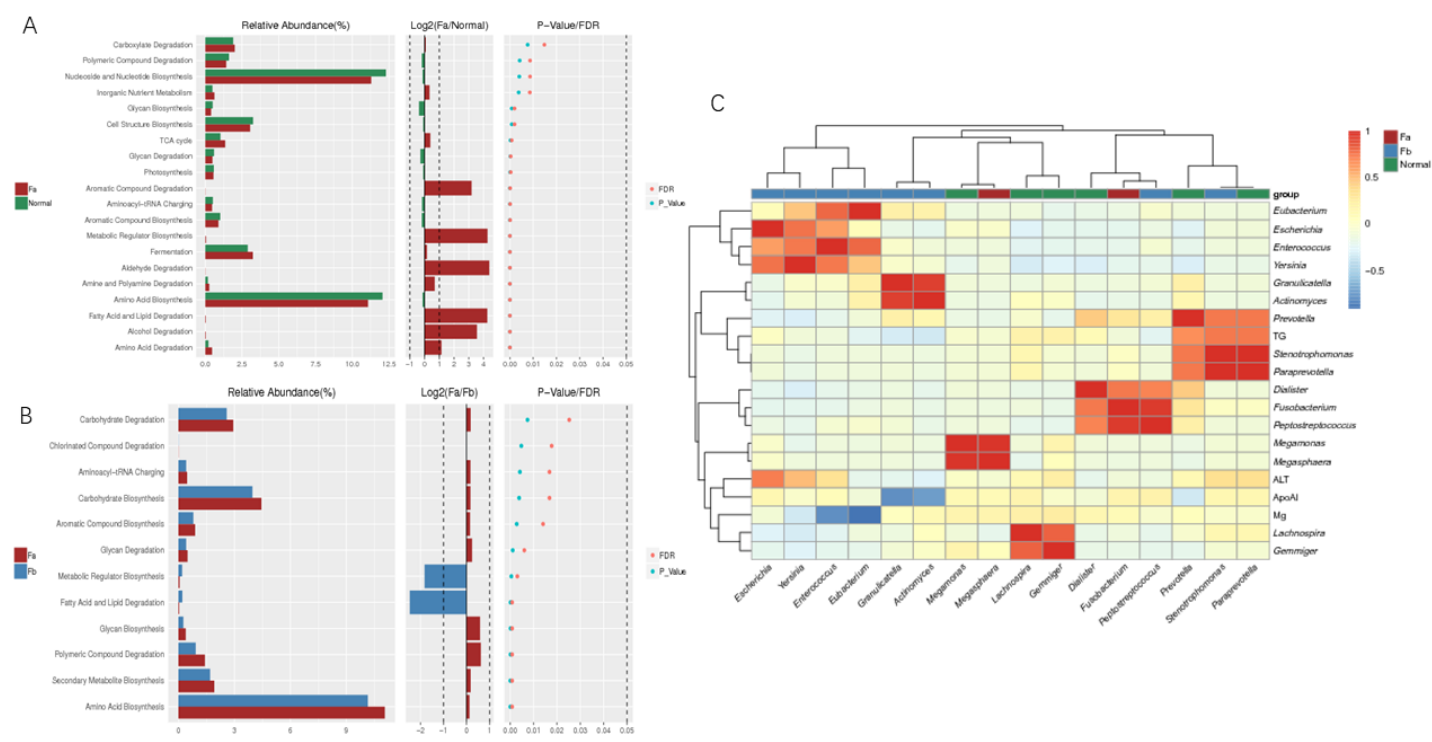

Figure 4. (A) Comparison of differential functional pathways in the Fa and Normal groups. correlation.

\section{Biomarkers and prognosis}

We followed-up 32 CRC patients who had provided pre- and post-treatment 

surgery). We evaluated the changes in important bacteria in paired stool samples. at the SUN YAT-SEN University Cancer Center after surgery, except for two cases of distinct increases. One CRC patient died after surgery, while the other had chronic enteritis (Figure 5A). Meanwhile, most patients treated at the SUN YAT-SEN Memorial Hospital exhibited the same decrease in Fusobacterium after surgery (Figure 5B). Five patients (A147, A119, B103, B106, B112) either developed postoperative recidivation or died. We observed that most of the samples showed an abnormal increase in Fusobacterium, and all of them exhibited an obvious decrease in beneficial bacteria (Faecalibacterium and Prevotella) sampling, such as one month after surgery, three months after surgery, and so on.

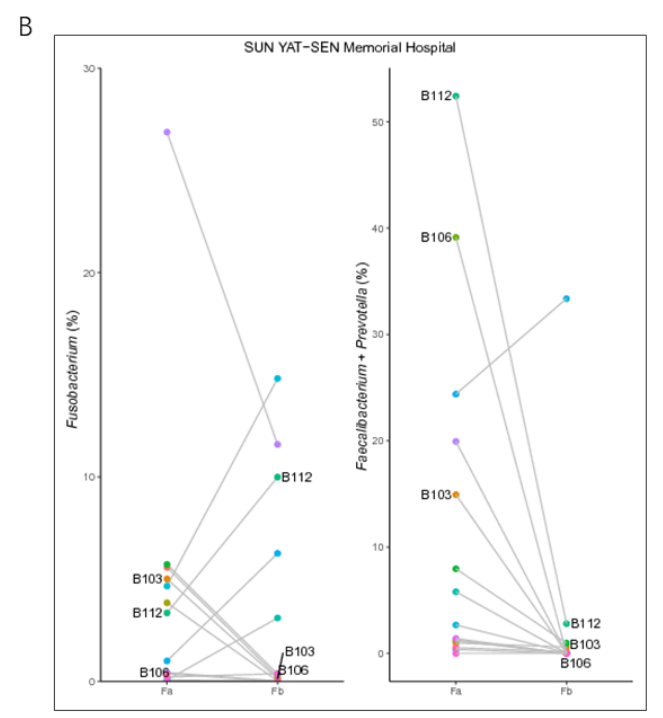

A

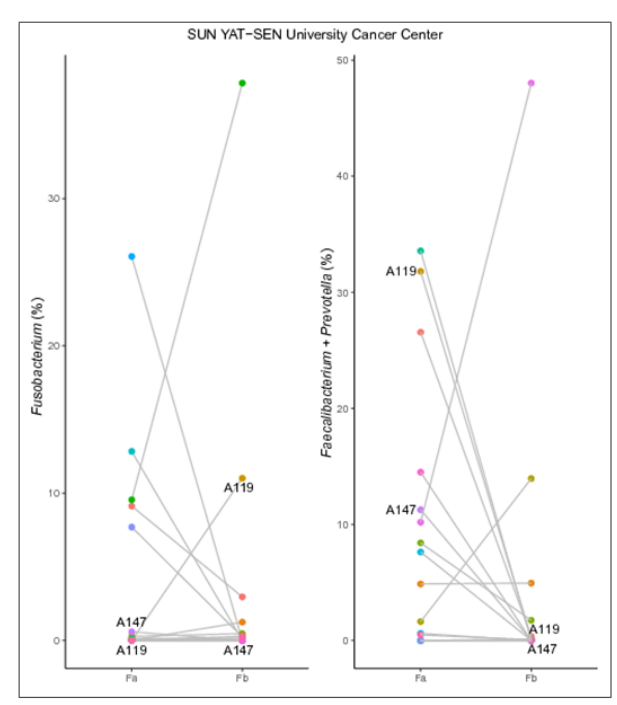




\section{Discussion}

In this study, we compared the fecal microbiota of CRC patients to those of healthy individuals and CRC patients that underwent surgery. We observed changes in the microbiota in all three groups. Moreover, the richness and biodiversity among these groups was found to differ. In particular, we found a decrease in biodiversity in the Fa group, and a strong decrease in biodiversity in wk after surgery), indicating that surgery might lead to serious microbiota dysbiosis. However, as patients in the $\mathrm{Fb}$ group were administered antibiotics after of Escherichia, Parabacteroides, and Fusobacterium being observed in both the

$310 \quad \mathrm{Fa}$ and $\mathrm{Fb}$ groups. Previous studies have reported a number of microbial species

311 found in CRC patients, most of which were present in our dataset as well. For instance, Fusobacterium was reported to coexist with tumors, and was considered 
313 to positively regulate tumor cell propagation ${ }^{12,13}$. F. nucleatum was demonstrated

314 to increase the tumor burden and selectively expand myeloid derived immune

315 cells, such as $\mathrm{CD} 11 \mathrm{~b}^{+}$, and myeloid derived suppressor cells in an $\mathrm{Apc}^{\mathrm{Min} /+}$ mouse

316 mode $1^{10}$. Other studies suggested that through the recruitment of tumor-infiltrating

317 immune cells, Fusobacteria might generate a proinflammatory microenvironment

318 that is conducive for colorectal neoplasia progression. In accordance with these

319 findings, our results showed that Fusobacterium was identified to be the principal

320 genus in the Fa group. Moreover, the classification model between the Fa and

321 Normal groups was credible with an AUC of 0.852, suggesting that

322 Fusobacterium could be used as a potential biomarker for CRC patients. Thus, the

323 increased abundance of Fusobacterium could be linked with a high risk of CRC.

324 Enterotoxigenic B. fragilis has been identified as a potential driver of CRC in both

325 human and mouse studies ${ }^{18,19,20}$. The toxin of $B$. fragilis is known to cause human

326 inflammatory diarrhea. However, it can also asymptomatically colonize a

327 proportion of the human population, thereby triggering colitis and strongly

328 inducing colonic tumors via activation of the T-helper type $17 \mathrm{~T}$-cell responses ${ }^{20}$.

329 In our dataset, we found that the species of B. fragilis was prominent in CRC

330 patients either before or after surgery, especially in the $\mathrm{Fb}$ group. E. coli is a

331 commensal bacterium of the human gut microbiota, but some pathogenic strains

332 have acquired the ability to induce chronic inflammation or produce toxins, such

333 as cyclomodulins, which could participate in carcinogenesis processes ${ }^{1,21,22}$. We

334 also tested the enrichment of Escherichia in CRC patients, especially in the $\mathrm{Fb}$ 
group. At the species level, the presence of E. coli was shown to be obviously

336 increased in the $\mathrm{Fa}$ and $\mathrm{Fb}$ groups than in the Normal group. All the

337 aforementioned bacteria involved in $\mathrm{CRC}$ are known to be

338 proinflammatory-associated, and hence might colonize faster in an inflammatory

339 conducive environment. As the fecal samples of the $\mathrm{Fb}$ group in this study were

340 obtained from CRC patients who had recently undergone surgery, their intestinal

341 microenvironments were probably unstable, with some potentially exhibiting an

342 inflammatory response, and some may have bad prognosis. Therefore, our

343 findings that the abundance of $B$. fragilis and E. coli was the largest in the $\mathrm{Fb}$

344 group, whereas Fusobacterium showed a slight decrease compared with that in the

$345 \quad$ Fa group, was justified. The distribution trend of these bacteria after surgery

346 should be analyzed in different stages post-surgery, and thus further research is

347 needed.

$348 \quad F a$. prausnitzii is one of the most abundant bacteria in the human intestinal

349 microbiota of healthy individuals, and the most important butyrate-producing

350 bacteria in the human colon $^{23}$, representing more than $5 \%$ of the total bacterial

351 population ${ }^{16}$. Further, this bacterium has shown potential to function as a probiotic

352 in the treatment of Crohn's disease ${ }^{24}$. Changes in the abundance of Fa. prausnitzii

353 have been linked to dysbiosis in several human disorders. To date, this commensal

354 bacterium has been considered as a bioindicator of human health. Prevotella, a

355 commensal bacterial genus known to produce short chain fatty acids and that

356 possesses potent anti-inflammatory effects, has been reported to be more 

who recrudesced or died after surgery.

The genus Enterococcus is of great relevance to human health because of its was justified.

\section{Conclusions}

In conclusion, this study showed the different composition of fecal microbiota among Chinese healthy individuals and CRC patients before and after surgery. We also identified Fusobacterium as a potential biomarker for CRC screening. We also found changes in the numbers of Fusobacterium, 
380 after surgery. This finding could contribute to CRC early screening and prognosis

381 monitoring in Chinese or Asian populations, in combination with multiple factors

382 of cfDNA methylation and alteration.

\section{Materials and Methods}

\section{Samples}

CRC patients were selected from Sun Yat-sen University Cancer Center and undergone any treatment, such as radiotherapy or chemotherapy, before use of antibiotics 1 mo prior to hospitalization. The cancer stage was identified according to the TNM classification of malignant tumors. CRC patients were first enrolled and hospitalized; then, their fecal samples were collected before surgery (approximately 2-3 d before surgery). CRC patients underwent operative

397 collection of their fecal samples approximately $1 \mathrm{wk}$ after resection surgery, they

398 were allowed to leave the hospital. Normal samples were collected from healthy 399 individuals with no history of cancer, chronic enteritis, chronic constipation, $400 \quad$ bloody stools, chronic appendicitis, or chronic cholecystitis. 

recorded (Table S4). of CRC patients (pre- and post-surgery corresponding to 72 stool samples) were collected, including biochemical criteria and routine blood examinations.

\section{Sample preparation and genomic DNA extraction}

None of the patients were subjected to any invasive operation, such as 
423 ethanol and resuspended in $1 \times$ TE buffer. DNA integrity and purification were

424 detected by agarose gel electrophoresis (1\%, $150 \mathrm{~V}, 40 \mathrm{~min})$.

Library construction and next generation sequencing (NGS)

Qualified samples were used for the library preparation process. The microbiota DNA was amplified by polymerase chain reaction (PCR) with a bacterial 16S rDNA V4 region universe primer pair ${ }^{5}$ 515F: quantitative PCR (QPCR).

\section{Sequence processing}

Raw sequences were assigned to each sample based on their unique barcode 
445 time, paired-end low-quality reads were filtered based on quality score, adapter

$446 \quad$ contamination, and $\mathrm{N}$ base ratio.

447 Paired-end clean reads were merged using FLASH (fast length adjustment of 448 short reads, v1.2.11 $)^{29}$ according to the relationship of the overlap between 449 paired-end reads. This was done when at least $15 \mathrm{bp}$ of the read overlapped the 450 read generated from the opposite end of the same DNA fragment, the maximum 451 allowable error ratio of an overlap region was set as 0.1 , and merged sequences were called clean tags.

Tags were assigned to OTUs using USEARCH (v7.0.1090) software $^{30}$, and 454 tags with $\geq 97 \%$ similarity were clustered to the same OTU. It has been reported 455 that a singleton OTU could be obtained due to sequencing errors or chimeras 456 generated during PCR; therefore, chimeric sequences were detected and removed 457 using UCHIME (v4.2.40) $)^{31}$ according to the match of representative OTUs to the 458 gold database (v20110519). The abundance of each OTU was quantified using 459 usearch_global algorithm by matching all clean tags to final OTUs, and 460 normalized using a standard number corresponding to the sample with the least $461 \quad$ sequences.

Representative OTUs were annotated using the RDP classifier (v2.2)

463 software ${ }^{32}$ based on the homolog of the Greengene database (v201305), with the 464 confidence threshold set to 0.8 . OTUs without annotation or annotated to polluted 465 species were removed, and the number of effective tags and information regarding 466 OTU taxonomic synthesis were recorded in a table for the next analysis. The 

taxonomy, with the relative abundance less than $0.5 \%$ in all samples combined

469 with others.

Common and specific OTUs among groups were compared and displayed using VennDiagram R (v3.1.1). Analysis of similarities were performed using Bray-Curtis in the vegan package of $\mathrm{R}$ (v3.5.1); comparison of differences between and within the groups was available, thereby allowing testing of the availability of grouping.

Alpha diversity was applied to analyze the complexity of species diversity of a sample using many indexes, such as observed species, Chao, Ace, Shannon, and Simpson. All indices of our samples were calculated using Mother (v1.31.2) ${ }^{33}$, and comparisons among groups were performed using the Kruskal test. Observed species and Chao were selected to identify community richness, whereas Shannon was used to identify community diversity. Beta diversity ${ }^{34,35}$ was used to evaluate

483 the differences in species complexity among different samples, and was calculated 484 on both weighted and unweighted UniFrac using QIIME (v1.80). Partial least 485 squares discrimination analysis (PLS-DA) was built using the mixOmics library of $486 \quad \mathrm{R}$ (v3.2.1), which was used to estimate the classification of samples and assess the variation in study groups.

We analyzed the differential abundance at the phylum, class, order, family, 
$490 \quad$ LEfSe $^{36}$, with the P value less than 0.01 and an LDA score more than 2 being

491 considered significant. To quantify the effective size of the differential taxa, we

492 used the fold change of the mean relative abundance between groups.

493 Comparisons between probabilities, as well as overall differences in the mean

494 relative abundance of each taxon between the two groups were evaluated using a

495 paired Wilcoxon rank sum test. Comparisons among three or more groups were

496 performed using the Kruskal-Wallis test.

497 The ROC curve was used to assess the confidence level of the classification

498 model. Accordingly, ROC analysis and the AUC values were calculated using the

$499 \quad$ pROC package of R.

500 MetaCyc pathway prediction was performed using Picrust2, MetaCyc

501 (https://metacyc.org/) containing pathways involved in primary and secondary

502 metabolism, related metabolites, and enzymatic reactions. Differential functions

503 were analyzed using the Wilcox-test between the two groups. Correlation was

504 tested by Pearson's coefficient using the R package.

506 Abbreviations:

507 CRC: colorectal cancer

508 ROS: reactive oxidative species

509 OTUs: operational taxonomic units

510 LDA: linear discriminant analysis 
511 LEfSe: linear discriminant analysis effect size

512 ROC: receiver operating characteristic

513 AUC: area under the ROC curve

514 NGS: next generation sequencing

515 PCR: polymerase chain reaction

516 QPCR: quantitative PCR

517 FLASH: fast length adjustment of short reads

518 PLS-DA: partial least squares discrimination analysis

519 ALT: alanine transaminase

520 ApoA1: apolipoprotein A1

521 TG: triglyceride

Acknowledgements

524 We acknowledge the volunteers who participated in our study.

526 Authors' contributions

527 Qiulin Yao: Methodology, Data curation, Formal analysis, Visualization,

528 Writing-Original draft preparation. Meifang Tang: Conceptualization, Methodology,

529 Supervision, Writing-Review \& Editing. Liuhong Zeng: Methodology, Selecting

530 samples, Communication. Zhonghua Chu: Conceptualization, Resources. Hui Sheng:

531 Resources. Yuyu Zhang: Resources. Yuan Zhou: Library preparation. Hongyun Zhang:

532 Review. Huayan Jiang: Providing information. Mingzhi Ye: Funding acquisition, 


\section{Funding}

536 This research was supported by the Guangzhou Science and Technology Plan Projects

537 (Health Medical Collaborative Innovation Program of Guangzhou) (grant No.

Trans-Omics Research (GZ2012, NO348).

\section{Availability of data and materials}

542 The data reported in this study are also available in the CNGB Nucleotide Sequence

543 Archive (CNSA: https://db.cngb.org/cnsa; accession number CNP0001385).

\section{Ethics approval and consent to participate}

546 The study was approved by appropriate Institutional Review Boards (IRB) of the BGI

547 (NO. BGI-IRB15100-T1).

\section{Consent for publication}

$550 \quad$ Not applicable.

\section{Competing Interests}

553 Authors declare no conflicts of interests. 


\section{Author details}

$556{ }^{1}$ Clinical laboratory of BGI Health, BGI-Shenzhen, Shenzhen 518083, China. ${ }^{2}$ BGI

557 Education Center, University of Chinese Academy of Sciences, Shenzhen 518083,

558 China. ${ }^{3}$ Guangdong Provincial Key Laboratory of Malignant Tumor Epigenetics and

559 Gene Regulation, Department of Gastrointestinal Surgery, Sun Yat-sen Memorial

560 Hospital, Sun Yat-sen University, Guangzhou 510060, China. ${ }^{4}$ Department of

561 Experimental Research, State Key Laboratory of Oncology in South China,

562 Collaborative Innovation Center for Cancer Medicine, Sun Yat-Sen University Cancer

563 Center, Guangzhou 510060, China. ${ }^{5}$ BGI Genomics, BGI-Shenzhen, Shenzhen

564 518083, China. ${ }^{6}$ BGI-Guangzhou Medical Laboratory, BGI-Shenzhen, Guangzhou

565 510006, China

566

\section{References}

568 [1] Bonnet M, Buc E, Sauvanet P, Darcha C, Dubois D, Pereira B, et al. Colonization

569 of the human gut by E. coli and colorectal cancer risk. Clin Cancer Res. 2014;20:859-

570 67. doi:10.1158/1078-0432.CCR-13-1343.

571 [2] Brennan CA, Garrett WS. Gut Microbiota, Inflammation, and Colorectal Cancer.

572 Annu Rev Microbiol. 2016;70:395-411. doi:10.1146/annurev-micro-102215-095513.

573 [3] O'Connell JB, Maggard MA, Liu JH, Etzioni DA, Ko CY. Are survival rates

574 different for young and older patients with rectal cancer? Dis Colon Rectum.

$575 \quad 2004 ; 47: 2064-9$.

576 [4] Collins D, Hogan AM, Winter DC. Microbial and viral pathogens in colorectal 
578 [5] Arthur JC, Perez-Chanona E, Muhlbauer M, Tomkovich S, Uronis JM, Fan TJ, et

579 al. Intestinal inflammation targets cancer-inducing activity of the microbiota. Science.

$580 \quad 2012 ; 338: 120-3$.

581 [6] Mathias LR, Giuseppina L, Bruno L, et al. Mucosa-associated microbiota 582 dysbiosis in colitis associated cancer. Gut Microbes. 2018;2:131-42.

583 [7] Cuevas-Ramos G, Petit CR, Marcq I, Boury M, Oswald E, Nougayrede J-P.

584 Escherichia coli induces DNA damage in vivo and triggers genomic instability in

585 mammalian cells. Proc Natl Acad Sci USA. 2010;107:11537-42.

586 [8] Wang X, Allen TD, May RJ, Lightfoot S, Houchen CW, Huycke MM.

587 Enterococcus faecalis induces aneuploidy and tetraploidy in colonic epithelial cells 588 through a bystander effect. Cancer Res. 2008;68:9909-17.

589 [9] Wang X, Yang Y, Moore DR, Nimmo SL, Lightfoot SA, Huycke MM. 590 4-hydroxy-2-nonenal mediates genotoxicity and bystander effects caused by 591 Enterococcus faecalis-infected macrophages. Gastroenterology. 2012;142:543-47.

592 [10] Kostic AD, Chun E, Robertson L, Glickman JN, Gallini CA, Michaud M, et al. 593 Fusobacterium nucleatum potentiates intestinal tumorigenesis and modulates the 594 tumor-immune microenvironment. Cell Host Microbe. 2013;14: 207-15.

595 [11] Gao R, Kong C, Huang L, Li H, Qu X, Liu Z, et al. Mucosa-associated 596 microbiota signature in colorectal cancer. Eur J Clin Microbiol Infect Dis. 2017; doi:10.1007/s10096-017-3026-4.

598 [12] Zackular JP, Rogers MA, Ruffin MT 4th, Schloss PD. The Human Gut 
601 [13] Mima K, Cao Y, Chan AT, Qian ZR, Nowak JA, Masugi Y, et al. Fusobacterium

602 nucleatum in Colorectal Carcinoma Tissue According to Tumor Location. Clin Transl

603 Gastroenterol. 2016;7:e200. doi:10.1038/ctg.2016.53.

604 [14] Zeller G, Tap J, Voigt AY, Sunagawa S, Kultima JR, Costea PI, et al. Potential of

605 fecal microbiota for early-stage detection of colorectal cancer. Mol Syst Biol. 606 2014;10:766. doi:10.15252/msb.20145645.

607 [15] Mima K, Nishihara R, Qian ZR, Cao Y, Sukawa Y, Nowak JA, et al. 608 Fusobacterium nucleatum in colorectal carcinoma tissue and patient prognosis. Gut. 609 2016;65:1973-80. doi:10.1136/gutjnl-2015-310101.

610 [16] Miquel S, Martin R, Rossi O, Bermudez-Humaran LG, Chatel JM, Sokol H, et al.

611 Faecalibacterium prausnitzii and human intestinal health. Curr Opin Microbiol. 612 2013;16:255-61. doi:10.1016/j.mib.2013.06.003. PMID:23831042.

613 [17] Yasuda K, Oh K, Ren B, Tickle TL, Franzosa EA, Wachtman LM, et al. 614 Biogeography of the intestinal mucosal and lumenal microbiome in the rhesus 615 macaque. Cell Host Microbe. 2015;17:385-91.

616 [18] Geis AL, Fan H, Wu X, Wu S, Huso DL, Wolfe JL, et al. Regulatory T-cell 617 response to enterotoxigenic Bacteroides fragilis colonization triggers IL17-dependent 618 colon carcinogenesis. Cancer Discov. 2015;5:1098-1109.

619 [19] Goodwin AC, Destefano Shields CE, Wu S, Huso DL, Wu X, Murray-Stewart 620 TR, et al. Polyamine catabolism contributes to enterotoxigenic Bacteroides 
621 fragilis-induced colon tumorigenesis. Proc Natl Acad Sci USA. 2011;108:15354-9.

$622[20]$ Wu S, Rhee K-J, Albesiano E, Rabizadeh S, Wu X, Yen HR, et al. A human

623 colonic commensal promotes colon tumorigenesis via activation of $\mathrm{T}$ helper type $17 \mathrm{~T}$

624 cell responses. Nat. Med. 2009;15:1016-22.

625 [21] Swidsinski A, Khilkin M, Kerjaschki D, Schreiber S, Ortner M, Weber J, et al.

626 Association between intraepithelial Escherichia coli and colorectal cancer.

627 Gastroenterology. 1998;115:281-6.

628 [22] Martin HM, Campbell BJ, Hart CA, Mpofu C, Nayar M, Singh R, et al.

629 Enhanced Escherichia coli adherence and invasion in Crohn's disease and colon

630 cancer. Gastroenterology. 2004;127:80-93.

631 [23] Ferreira-Halder CV, Faria AVS, Andrade SS. Action and function of

632 Faecalibacterium prausnitzii in health and disease. Best Pract Res Clin Gastroenterol.

$6332017 ; 31: 643-8$.

634 [24] Sokol H, Pigneur B, Watterlot L, Lakhdari O, Bermúdez-Humarán LG,

635 Gratadoux JJ, et al. Faecalibacterium prausnitzii is an antiinflammatory commensal

636 bacterium identified by gut microbiota analysis of Crohn disease patients. Proc Natl

637 Acad Sci USA. 2008;105:16731-6.

638 [25] Martínez I, Stegen JC, Maldonado-Gómez MX, Eren AM, Siba PM, Greenhill

639 AR, et al. The gut microbiota of rural Papua New Guineans: Composition, diversity

640 patterns, and ecological processes. Cell Rep. 2015;11:527-38.

641 [26] Vuillermin PJ, O’Hely M, Collier F, Allen KJ, Tang MLK, Harrison LC, et al.

642 Maternal carriage of Prevotella during pregnancy associates with protection against 
643 food allergy in the offspring. Nat Commun. 2020;11:1452.

644 [27] Garcia-Solache M, Rice LB. The Enterococcus: a Model of Adaptability to Its

645 Environment. Clin Microbiol Rev. 2019;32:e00058-18. doi:10.1128/CMR.00058-18.

646 [28] Qin J, Li Y, Cai Z, Li S, Zhu J, Zhang F, et al. A metagenome-wide association

647 study of gut microbiota in type 2 diabetes. Nature. 2012;490:55-60.

648 [29] Magoc T, Salzberg S. FLASH: Fast length adjustment of short reads to improve

649 genome assemblies. Bioinformatics. 2011;27:2957-63.

650 [30] Edgar RC. Search and clustering orders of magnitude faster than BLAST.

651 Bioinformatics. 2010;26:2460-1. doi:10.1093/bioinformatics/btq461.

652 [31] Edgar RC, Haas BJ, Clemente JC, Quince C, Knight R. UCHIME improves

653 sensitivity and speed of chimera detection. Bioinformatics. 2011;27:2194-2200.

654 [32] Wang Q, Garrity GM, Tiedje JM, Cole JR. Naive Bayesian classifier for rapid 655 assignment of rRNA sequences into the new bacterial taxonomy. Appl Environ 656 Microbiol. 2007;73:5261-7.

657 [33] Patrick DS, Sarah LW, Ryabin T, Hall JR, Hartmann M, Hollister EB, et al. 658 Introducing mothur: Open-Source, Platform- Independent, Community-Supported 659 Software for Describing and Comparing Microbial Communities. Appl Environ 660 Microbiol. 2009;75:7537-41.

661 [34] Lozupone C, Lladser ME, Knights D, Stombaugh J, Knight R. UniFrac: an 662 effective distance metric for microbial community comparison. ISME J. 2011;5:169663 72.

664 [35] Lozupone C, Hamady M, Kelley ST, Knight R. Quantitative and qualitative $\beta$ 
665 diversity measures lead to different insights into factors that structure microbial

666 communities. Appl Environ Microbiol. 2007;73:1576-85.

667 [36] Segata N, Izard J, Waldron L, Gevers D, Miropolsky L, Garrett WS, et al.

668 Metagenomic biomarker discovery and explanation. Genome Biol. 2011;12:R60.

669 